\title{
Vector Affine Quantization Can Create Valid Quantum Field Theories
}

\author{
John R. Klauder \\ Department of Physics and Department of Mathematics, University of Florida, Gainesville, USA \\ Email: klauder@ufl.edu
}

How to cite this paper: Klauder, J.R. (2022) Vector Affine Quantization Can Create Valid Quantum Field Theories. Journal of High Energy Physics, Gravitation and Cosmology, 8, 237-242.

https://doi.org/10.4236/jhepgc.2022.81017

Received: December 13, 2021

Accepted: January 22, 2022

Published: January 25, 2022

Copyright (c) 2022 by author(s) and Scientific Research Publishing Inc. This work is licensed under the Creative Commons Attribution International License (CC BY 4.0).

http://creativecommons.org/licenses/by/4.0/

\begin{abstract}
Affine quantization, a parallel procedure to canonical quantization, needs to use its principal quantum operators, specifically $D=(P Q+Q P) / 2$ and $Q \neq 0$, to represent appropriate kinetic factors, such as $P^{2}$, which involves only one canonical quantum operator. The need for this requirement stems from path integral quantizations of selected problems that affine quantization can solve but canonical quantization fails to solve. This task is resolved for simple examples, as well as examples that involve scalar, and vector, quantum field theories.
\end{abstract}

\section{Keywords}

Affine Quantization, Vector Field Models, Flexibility of the Dilation Variable

\section{Elements of Basic Affine Quantization}

Affine quantization (AQ) has been created from canonical quantization (CQ) in the sense that from $P$ and $Q$, which obey $[Q, P]=i \hbar 1$, we build the dilation operator $D \equiv\left(P^{\dagger} Q+Q P\right) / 2$ along with $Q \neq 0$, stated otherwise as $Q^{2}>0$, which leads to $[Q, D]=i \hbar Q$. The reason that $Q \neq 0$ is that if $Q=0$ then $D=0$ and $P$ cannot help. Because $Q$ is "incomplete", then $P^{\dagger} \neq P$. However, $P^{\dagger} Q=P Q$, thanks to $Q \neq 0$. And so $D=(P Q+Q P) / 2$ as well.

The dilation operator can be chosen in different ways that help formulate and solve various problems. In particular, if $D=[P F(Q)+F(Q) P] / 2$ along with $F(Q) \neq 0$, it follows, that $[F(Q), D]=i \hbar\left(F(Q)^{2}\right)^{\prime} / 2$, wherein the prime signals a differentiation with respect to $Q$.

An interesting way in which AQ can lead to CQ arises in the partial-harmonic oscillator story. Using Schrödinger's representation, the Hamiltonian operator is 
given by $\left[-\hbar^{2} d^{2} / \mathrm{d} x^{2}+(3 / 4) \hbar^{2} /(x+b)^{2}+x^{2}\right] / 2$ in which $b \geq 0$ and $x>-b$, with $b=0$ representing the half-harmonic oscillator. While the eigenvalues of the full harmonic oscillator are $\hbar[(0,1,2, \cdots)+1 / 2]$, the eigenvalues of the half-harmonic oscillator are $2 \hbar[(0,1,2, \cdots)+1]$, and both sets of these eigenvalues are equally spaced, as are the eigenvalues, with different separations for different values of $b$. While this model belongs to AQ for all finite $b$, AQ passes to CQ when $b \rightarrow \infty$ [1] [2] [3].

Recently, the affine version of the kinetic operator $P^{2}$, which is $D G(Q)^{2} D$, with $G(Q)=1 / F(Q)$, was analyzed. It was shown that it led to a different correction term which is proportional to $\hbar^{2}$. While the classical Hamiltonian for the harmonic oscillator is $\left(p^{2}+q^{2}\right) / 2$, with $-\infty<p, q<\infty$, the half-harmonic oscillator requires that $q>0$. The quantization of the half-harmonic oscillator fails using CQ, but succeeds using AQ. The additional factor provided by $D G^{2} D$ led to the correct quantization of the half-harmonic oscillator [1] [2]. While the classical Hamiltonian for a single harmonic oscillator can be given by $\left(p^{2}+q^{2}\right) / 2$, where $p$ represents momentum and $q$ represents position, it can also represent several classical harmonic oscillators. All that is necessary is to let $p^{2} \Rightarrow p_{1}^{2}+p_{2}^{2}+\cdots=\vec{p}^{2}$ and $q^{2} \Rightarrow q_{1}^{2}+q_{2}^{2}+\cdots=\vec{q}^{2}$. The same story applies CQ to the quantum Hamiltonian of a single harmonic oscillator $\left(P^{2}+Q^{2}\right) / 2$, and it becomes many oscillators, $\left(P^{2}+Q^{2}\right) / 2 \Rightarrow\left(\vec{P}^{2}+\vec{Q}^{2}\right) / 2$. Now, for the half-harmonic oscillator, the classical Hamiltonian is $\left(p^{2}+q^{2}\right) / 2$ with $q>0$, and the quantum Hamiltonian is given by $\mathcal{H}=\left[P^{2}+(3 / 4) \hbar^{2} / Q^{2}+Q^{2}\right] / 2$, with $Q>0$. The vector version, which can retain rotational symmetry of this quantum Hamiltonian, would be $\mathcal{H}=\left[\vec{P}^{2}+(3 / 4) \hbar^{2} / \vec{Q}^{2}+\vec{Q}^{2}\right] / 2$, with $\vec{Q}^{2}>0$. In addition, this expression can also require that every oscillator has only positive coordinates. For a two-component vector, we can imagine that accepted vectors resemble a clock's minute hand which only points between 12 noon and $3 \mathrm{pm}$.

\section{Scalar Field Models That Lead to Vector Field Models}

\subsection{A Scalar Model That Is Familiar}

Our first model has a classical Hamiltonian given by

$$
H=\int\left\{\frac{1}{2}\left[\pi(x)^{2}+(\vec{\nabla} \varphi(x))^{2}+m^{2} \varphi(x)^{2}\right]+g \varphi(x)^{p}\right\} d^{s} x,
$$

where $p=4,6,8, \cdots$ is the interaction power, and $n=s+1$ is the number of spacetime coordinates. The affine dilation variable is $\kappa(x)=\pi(x) \varphi(x)$ with $\varphi(x) \neq 0$. It follows that the classical Hamiltonian in affine variables is given by

$$
H=\int\left\{\frac{1}{2}\left[\kappa(x)^{2} / \varphi(x)^{2}+(\vec{\nabla} \varphi(x))^{2}+m^{2} \varphi(x)^{2}\right]+g \varphi(x)^{p}\right\} \mathrm{d}^{s} x,
$$

in which $0<\varphi(x)^{-2}<\infty$ now to protect $\kappa(x)$, and it all leads to require that $0<|\varphi(x)|^{p}<\infty$. By just using $A Q$ variables, instead of CQ variables, it is noteworthy that Equation (2) has absolutely eliminated any non-renormalizability!

Using AQ and Schrödinger's representation, the quantum Hamiltonian for 
this model is

$$
\mathcal{H}=\int\left\{\frac{1}{2}\left[\hat{\kappa}(x) \varphi(x)^{-2} \hat{\kappa}(x)+(\vec{\nabla} \varphi(x))^{2}+m^{2} \varphi(x)^{2}\right]+g \varphi(x)^{p}\right\} \mathrm{d}^{s} x,
$$

which, following Section 1, leads to a formal version of the affine quantum Hamiltonian,

$$
\begin{aligned}
\mathcal{H}= & \int\left\{\frac { 1 } { 2 } \left[\hat{\pi}(x)^{2}+(3 / 4) \delta^{2 s}(0) \hbar^{2} / \varphi(x)^{2}\right.\right. \\
& \left.\left.+(\vec{\nabla} \varphi(x))^{2}+m^{2} \varphi(x)^{2}\right]+g \varphi(x)^{p}\right\} \mathrm{d}^{s} x .
\end{aligned}
$$

At this point we introduce a paragraph from [4] that shows how to eliminate $\delta^{2 s}(0)$.

"The origin of $\delta^{s}(0)=\infty$ is simply due to the fact that $[\hat{\varphi}(x), \hat{\pi}(x)]=i \hbar \delta^{s}(0) 11$. In a sense, this result is unusual. For example, for a single classical variable $(p q)^{2}<\infty$ and $|Q P-P Q|^{2}=\hbar^{2} 11$. However, for a classical field $(\pi(x) \varphi(x))^{2}<\infty$ while $|\hat{\varphi}(x) \hat{\pi}(x)-\hat{\pi}(x) \hat{\varphi}(x)|^{2}=\infty \hbar^{2} \mathbb{1}$. When approximated, as for an integration, then $\hat{\varphi}(x) \rightarrow \hat{\varphi}_{k}$ and $\hat{\pi}(x) \rightarrow \hat{\pi}_{k}$, where instead of the continuum that $x$ represents, $k$ identifies different points on a discrete lattice. This leads to $\left[\hat{\varphi}_{k}, \hat{\pi}_{k}\right]=i \hbar a^{-s} 1$, where $a$ is a tiny spacial distance between neighboring lattice points. In preparation for an integration, just as every integral involves a continuum limit of an appropriate summation, these expressions are used in Monte Carlo (MC) calculations which involve proper sums for their 'integrals'. All of this is designed to provide a path integral quantization, and, when necessary, their sums need to be regularized. In our case, the regularized version becomes appropriately 'scaled': specifically $\varphi_{k} \rightarrow a^{-s / 2} \varphi_{k}$, $\pi_{k} \rightarrow a^{-s / 2} \pi_{k}, \quad \kappa_{k} \rightarrow a^{-s} \kappa_{k}, \quad g \rightarrow a^{s(p-2) / 2} g$, and the regularized $d^{s} x \rightarrow a^{s}$ may also be scaled as $a^{s} \rightarrow a^{2 s}$. After all, classical expressions do not admit $\delta(0)$ terms and path integration requires only classical expressions for their integrands."

In this paper, there will be other models that introduce $\delta^{2 s}(0)$ type divergences. The kind of procedures outlined in the foregoing paragraph can tackle any one of them.

\subsection{An Example in Which AQ Passes to CQ}

To illustrate this example with CQ, we first choose $n=s+1=3$ and $\varphi_{3}^{4}$ and $\varphi_{3}^{8}$ models, because the first model succeeds using CQ, while the second model fails using CQ. We start with AQ and a modified and regularized version of the quantum Hamiltonian, with Schrödinger's representation and $\varphi_{k} \neq 0$, so long as $0 \leq b<\infty$, and given by ${ }^{1}$

$$
\begin{aligned}
\mathcal{H}= & \sum_{k}\left\{\frac { 1 } { 2 } \left[\hat{\pi}_{k}^{2}+(3 / 4) \hbar^{2} /\left(\varphi_{k}^{2}\right)(b+1)+\sum_{k^{*}, k}\left(\varphi_{k^{*}}-\varphi_{k}\right)^{2} / a^{2}\right.\right. \\
& \left.\left.+m^{2} \varphi_{k}^{2}\right]+g\left|\varphi_{k}\right|^{(8+4 b) /(1+b)}\right\} a^{2},
\end{aligned}
$$

${ }^{1}$ The regularized sum in (5) has been scaled in which the factor $a^{-4}$ was removed from the " $3 / 4$ " term. 
which, with $b=0$, starts as the AQ model of $\varphi_{3}^{8}$, and when $b \rightarrow \infty$, it finally becomes the CQ model of $\varphi_{3}^{4}$. In this example, the absence of non-renormalizability, for any value of $b$, holds true.

\section{Two Valid Affine Quantizations of Vector Models}

Our first task will be to turn a scalar field model into a vector field model. The classical Hamiltonian in (1) is our first target. All that is necessary is to let $\pi(x)^{2} \Rightarrow \pi_{1}(x)^{2}+\pi_{2}(x)^{2}+\cdots=\vec{\pi}(x)^{2}$ and

$\varphi(x)^{2} \Rightarrow \varphi_{1}(x)^{2}+\varphi_{2}(x)+\cdots=\vec{\varphi}(x)^{2}$ for two of the scalar terms, and $(\vec{\nabla} \varphi(x))^{2}$ becomes $(\vec{\nabla} \cdot \vec{\varphi}(x))^{2}$.

\subsection{A Vector Model That Is Common}

The classical Hamiltonian for this vector model is

$$
H=\int\left\{\frac{1}{2}\left[\vec{\pi}(x)^{2}+(\vec{\nabla} \cdot \vec{\varphi}(x))^{2}+m^{2} \vec{\varphi}(x)^{2}\right]+g\left(\vec{\varphi}(x)^{2}\right)^{r}\right\} \mathrm{d}^{s} x .
$$

The term $n=s+1$ represents the number of spacetime dimensions as before, while now $r=2,3,4, \cdots$, is the interaction power. Such models can also fail with $\mathrm{CQ}$, and we will focus on AQ. The classical dilation variable now is $\kappa(x)=\vec{\pi}(x) \cdot \vec{\varphi}(x)$, with $\vec{\varphi}(x) \neq \mathbf{0}$, or stated differently, $\vec{\varphi}(x)^{2}>0$. The quantum Hamiltonian, expressed in affine variables and in Schrödinger's representation, is given by

$$
\mathcal{H}=\int\left\{\frac{1}{2}\left[\hat{\kappa}(x)\left(\vec{\varphi}(x)^{2}\right)^{-1} \hat{\kappa}(x)+(\vec{\nabla} \cdot \vec{\varphi}(x))^{2}+m^{2} \vec{\varphi}(x)^{2}\right]+g\left(\vec{\varphi}(x)^{2}\right)^{r}\right\} \mathrm{d}^{s} x .
$$

In this case the kinetic factor, again with Schrödinger's representation, becomes

$$
\hat{\kappa}(x)\left(\vec{\varphi}(x)^{2}\right)^{-1} \hat{\kappa}(x)=\hat{\vec{\pi}}(x)^{2}+(3 / 4) \hbar^{2} \delta^{2 s}(0) /\left(\vec{\varphi}(x)^{2}\right),
$$

and once again a scaled version to eliminate $\delta^{2 s}(0)$ is readily obtained. By choice, this vector model exhibits full rotational symmetry.

\subsection{A Vector Model That Is Less Common}

The classical Hamiltonian, expressed in canonical variables, is given by

$$
H=\int\left\{\frac{1}{2}\left[\vec{\pi}(x)^{2}+(\vec{\nabla} \cdot \vec{\varphi}(x))^{2}+m^{2} \vec{\varphi}(x)^{2}\right]+g\left(\vec{\varphi}(x)^{2}-\Phi^{2}\right)^{r}\right\} d^{s} x,
$$

where now $r=2,4,6, \cdots$. The dilation variable is $\kappa(x)=|\vec{\pi}(x)|\left(\vec{\varphi}(x)^{2}-\Phi^{2}\right)$, where $|\vec{\pi}(x)|^{2}=\vec{\pi}(x)^{2}$, and with $\left(\vec{\varphi}(x)^{2}-\Phi^{2}\right) \neq 0$. This leads to the classical Hamiltonian, now expressed in affine variables, which is given by

$$
\begin{aligned}
H= & \int\left\{\frac{1}{2}\left[\kappa(x)^{2} /\left(\vec{\varphi}(x)^{2}-\Phi^{2}\right)^{2}+(\vec{\nabla} \cdot \vec{\varphi}(x))^{2}+m^{2} \vec{\varphi}(x)^{2}\right]\right. \\
& \left.+g\left(\vec{\varphi}(x)^{2}-\Phi^{2}\right)^{r}\right\} \mathrm{d}^{s} x,
\end{aligned}
$$


and, as before, this expression has no non-renormalization. While $\left(\vec{\varphi}(x)^{2}-\Phi^{2}\right) \neq 0$ divides the field $\vec{\varphi}(x)$, the presence of the gradient term enforces continuity of $\vec{\varphi}(x)$ between regions.

Adopting Schrödinger's representation, the affine quantum Hamiltonian is given by

$$
\begin{aligned}
\mathcal{H}= & \int\left\{\frac{1}{2}\left[\hat{\kappa}(x)\left(\vec{\varphi}(x)^{2}-\Phi^{2}\right)^{-2} \hat{\kappa}(x)+(\vec{\nabla} \cdot \vec{\varphi}(x))^{2}+m^{2} \vec{\varphi}(x)^{2}\right]\right. \\
& \left.+g\left(\vec{\varphi}(x)^{2}-\Phi^{2}\right)^{r}\right\} \mathrm{d}^{s} x,
\end{aligned}
$$

and guided by the analysis leading to Equation (10) in [4], it would show that the kinematic variable becomes

$$
\begin{aligned}
& \hat{\kappa}(x)\left(\vec{\varphi}(x)^{2}-\Phi^{2}\right)^{-2} \hat{\kappa}(x) \\
& =\hat{\pi}(x)^{2}+\hbar^{2} \delta^{2 s}(0)\left(2 \vec{\varphi}(x)^{2}+\Phi^{2}\right) /\left(\vec{\varphi}(x)^{2}-\Phi^{2}\right)^{2} .
\end{aligned}
$$

Once again, scaled regularization can remove the factor $\delta^{2 s}(0)$ from this expression. Another story of passing from AQ to CQ could be devised for this model along the same lines as in Section 2.2, but that would not add to any physical understanding.

\section{Summary}

The application of CQ to quantum field theory has had successful results along with its share of unsolved issues. This paper is focused on the use of AQ in quantizing several typical quantum field models for which CQ has either not yet solved or has solved but with unsatisfactory results. The AQ examples discussed in this paper may be able to offer valuable assistance to the challenges that face quantum field theory.

It is important to understand that AQ does not replace CQ, but instead, AQ joins together with CQ in what this author has declared it to be, namely, Enhanced Quantization, or EQ for short [5]!

\section{Conflicts of Interest}

The author declares no conflicts of interest regarding the publication of this paper.

\section{References}

[1] Klauder, J. (2020) Quantum Gravity Made Easy. Journal of High Energy Physics, Gravitation and Cosmology, 6, 90-102. https://doi.org/10.4236/jhepgc.2020.61009

[2] Gouba, L. (2021) Affine Quantization on the Half Line. Journal of High Energy Physics, Gravitation and Cosmology, 7, 352-365. https://doi.org/10.4236/jhepgc.2021.71019 https://arxiv.org/abs/2005.08696

[3] Handy, C.R. and Klauder, J. (2021) Proof that Half-Harmonic Oscillators Become Full-Harmonic Oscillators after the "Wall Slides Away". arXiv:2108.00289. 
[4] Fantoni, R. and Klauder, J. (2021) Kinetic Factors in Affine Quantization and Their Role in Field Theory Monte Carlo. Submitted for Publication.

[5] Klauder, J. (2015) Enhanced Quantization: Particles, Fields \& Gravity. World Scientific, Singapore. https://doi.org/10.1142/9452 